\title{
Profile of Urinary Tract Infection in Indwelling Catheterized Patients
}

\author{
Dr. S. G.Kulkarni ${ }^{1}$, Dr. S. H. Talib ${ }^{2}$, Dr. Manjiri Naik ${ }^{3}$, Dr. Amit Kale ${ }^{4}$ \\ ${ }^{1}$ Professor \& Head Department of Nephrology, ${ }^{2}$ Professor \& Head Department of Medicine, ${ }^{3}$ M.D. (General \\ Medicine) Associate Professor,${ }^{4}$ Chief Resident in Medicine,M.G.M. Medical College \& Hospital, Aurangabad \\ 431003.
}

\begin{abstract}
The urinary tract is the most common site of nosocomial infection and most of these infections follow instrumentation of the urinary tract, mainly urinary catheterization and is a frequent cause of significant morbidity, sepsis and death. Hence this study was done to evaluate the incidence of CATHETER ASSOCIATED Urinary tract infection (CAUTI) admitted in intensive care unit (ICU) of MGM MEDICAL COLLEGE \& HOSPITAL AURANGABAD from July 2011 to September 2013.
\end{abstract}

Keywords: Nosocomial infection, Sepsis, Catheter associated urinary tract infection.

\section{Introduction}

The urinary tract is the most common site of nosocomial infection and most of these infections follow instrumentation of the urinary tract, mainly urinary catheterization and is a frequent cause of significant morbidity, sepsis and death. Most frequently bacteria from the urethral meatus ascend to the bladder between the catheter and urethral surfaces. Alternatively, bacteria may ascend with in the urine drainage systems following contamination of the drainage bag or catheter tubing junction. The presence of bacteria in the bladder constitutes a potential reservoir for multi resistant bacteria. ${ }^{7}$ The risk of acquiring a urinary tract infection depends on the method and duration of catheterization, and the quality of catheter care. Catheter associated bacteriuria is defined as a quantitative culture with $>10^{5}$ organisms $/ \mathrm{ml}$ with not more than two different species of organisms. ${ }^{8}$ Bacteriuria develops in at least $10-15 \%$ of hospitalized patients with indwelling urethral catheters. The risk of infection is almost 3-10\% per day of catheterization. E.coli, Proteus, Pseudomonas, Klebsiella, Serratia, Staphylococci, Enterococci, and Candida are the pathogens that usually cause these infections. ${ }^{6}$ Factors associated with an increased risk of catheter- associated UTI include female sex, prolonged catheterization, severe underlying illness, disconnection of the catheter and drainage tube, other types of faculty catheter care, and lack of systemic antimicrobial therapy. ${ }^{6}$

So in the present study, we aimed to determine the incidence and pattern of catheter associated urinary tract infection (CAUTI). This gives health care providers a comprehensive understanding of the institutional problems, confronting them, and a way to resolve these problems.

\section{Material And Methods}

The present study "Incidence and Pattern of Catheter Associated Urinary Tract Infection in Patients Admitted in all patients admitted to ICU in medical college hospital in marathwada region Maharashtra during the period from July 2011 to September 2013. Duration of study: 28 months. Study design: Prospective Nonrandomized Study. No of Patients: 204. Method used for statistics: Chi Square test and P value. The data was gathered according to the definitions provided by the CDC. A detailed history of patient and through clinical examination of the patient was done.

Inclusion Criteria: All patients admitted in MGM Hospital in ICU and catheterized of Age above the 18 years. Exclusion Criteria: Benign enlargement of prostate, already catheterized patients, Pregnancy, Diagnosed case of urinary tract infection with previous urine culture \& sensitivity positive.

\section{Observations}

The present study comprised of 204 patients who were catheterized in ICU from July 2011 September 2013.Out of them 44 patients developed CAUTI Hence, incidence of CAUTI during the study period figured to $21.47 \%$ (Table 1). The mean age of male population was 48.17 years with a SD of 14.34 years and that of female population was 46.23 years with a SD of 12.45 years $P$ value was $>0.5$ which was not significant (Table3). Out of 44 total CAUTI cases 30 patients were male and 14 were females. $68.18 \%$ of CA-UTI cases were males and $31.81 \%$ were female patients. $\mathrm{P}$ value came out to be $>0.05$, so not significant (Table4). Considering Age groups $>40$ years and $<40$ years in both males and females together in CAUTI $37(84 \%)$ out of 
44 were $>40 y e a r s$ age and $7(16 \%)$ out of 44 were $<40 y e a r s$ age, it was found to be statistically significant (p value < 0.01) (Table5). Out of 204 patient 33 were diabetic and out of them 13 patient (39\%) had CAUTI with P value $<0.01$ Significant (Table6). Out of 204 patient 44 were having Renal failure and out of them 22 patient (50\%) had CAUTI, with P value <0.001 Significant (Table7).15 patient (34.90\%) had CAUTI on Day 3 and 29 (65.09\%) having CAUTI on Day 7 out of 44 CAUTI patients (Table8).Five most common isolates from CAUTI cases were E.coli in $47.36 \%$ cases each, Klebsiella (19.20\%), Pseudomonas (14.10\%) and Candida in $8.70 \%$ cases(Table9). Amikacin is found to be the most sensitive antibiotic in CA-UTI cases in our study accounting for its susceptibility in $65.38 \%$ cases followed by Imepenem in $57.69 \%$ cases each. Followed by Piperacillin+Tazobactum $53.84 \%$ cases each in our study (Table10). Resistance was found mostly in Penicillins (92.30\%), Cephalosporins (90.38\%), and Fluroquinolones (69.23\%), cotrimoxazole (82.69\%) cases each in our study (Table11).

\section{Discussion}

The present study has been conducted on 204 catheterized patients, out of which 44 patients developed CAUTI. This result is comparable with those of $\mathrm{N}$ Bhatia et $\mathrm{al}^{11}$ (2010), Dr I Bagchi et al ${ }^{14}$ (2013), Yonit Wiener well et $\mathrm{al}^{9}$ (2013)

Occurrence of CAUTI was more in male patients than that of female patients and male predominance i.e 30 out of $44(68.18 \%)$ compared to females 14 out of $44(31.81 \%)$ was noted in CAUTI cases, although the association was not found to be significant. Comparable studies with similar male predominance like $\mathrm{N}$ Bhatia et $\mathrm{al}^{11}$, Joon Ho Lee et $\mathrm{al}^{12}$, Jaggi $\mathrm{N}$ etal ${ }^{17}$

The number of CAUTI cases increases with the age of the patient. Out of 44 CAUTI cases, the maximum incidence was from $>40$ years age group. Association was seen between age $>40$ years and age $<40$ years considering both males and females together in CAUTI; 37(84\%) out of 44 were >40years age and 7(16\%) out of 44 were $<40 y e a r s$ age. This was found to be statistically significant ( $\mathrm{p}$ value $<0.01$ ), which means that those aged $>40$ years were having the risk of developing CAUTI more than who were aged $<40$. This is comparable to studies with similar predominance of CAUTI in $>40$ years age like $\mathrm{N}$ Bhatia et $\mathrm{al}^{11}$, Joon Ho Lee et $\mathrm{al}^{12}$, Jaggi $\mathrm{N}$ et $\mathrm{al}^{17}$.

13 patients (39.09\%) out of 44 CAUTI cases had diabetes mellitus and $20(12.5 \%)$ out of 160 patients who were not having CAUTI had diabetes mellitus. When association was seen, it was found to be statistically significant

Many studies like our study which is comparable to the findings of the studies by Yonit Wiener well et $\mathrm{al}^{9}$, Joon Ho Lee et $\mathrm{al}^{12}$ and Dr I Bagchi et al ${ }^{14}$

Renal Failure had increased significant risk $\mathrm{p}$ value $<0.001$ which signifies that those with renal failure had more risk of developing CAUTI Similar findings of the studies by Yonit Wiener well et $\mathrm{al}^{9}, \mathrm{E}^{\mathrm{T}}$ Tissot et $\mathrm{al}^{10}$

patients were followed-up till 7 days of catheterization. Out of 44 cases, 15(34.09\%) were detected within 3 days of catheterization, $29(65.90 \%)$ were detected within 7 days of catheterization. This is comparable to findings of the studies done by N Bhatia et al ${ }^{11}$, Dr I Bagchi et al $^{14}$

E.coli was the commonest isolate (47.36\%), Klebsiella species amounting to19.2\%. Pseudomonas aeruginosa $(14.10 \%)$

Comparable to study conducted by Dr.I Bagchi et $\mathrm{al}^{14}$, Dogru et $\mathrm{al}^{18}$, Sallam SA et al ${ }^{17}$, N Bhatia et $\mathrm{al}^{11}$, Dimri sanjeev et $\mathrm{Al}^{13}$.

Klebsiella associated CAUTI was as the second most common organism in studies done by $\mathrm{N}$ Bhatia et $\mathrm{al}^{11}$, Dimri sanjeev et $\mathrm{al}^{13}$ and Dr.I Bagchi et $\mathrm{l}^{14}$ respectively, which is comparable to our study

Pseudomonas was seen as the third most commonly found isolate in our study which is comparable to findings seen in studies done by $\mathrm{N}$ Bhatia et $\mathrm{al}^{11}$, Dimri sanjeev et $\mathrm{al}^{13}$ and Dr.I Bagchi et al ${ }^{14}$.

The emergence of Candida infection with incidence of $8.7 \%$ in our study is also comparable to other studies such as the study done by $\mathrm{N}$ Bhatia et a ${ }^{11}$

CAUTI cases were most susceptible to the antibiotic Amikacin (65.38\% cases), Imipenem sensitivity in 57.69\% , Piperacillin+Tazobactum showed $53.84 \%$, Nitrofurantoin sensitivity was seen in $46.15 \%$, which is comparable to studies by Dimri sanjeev et $\mathrm{al}^{13}$, Dr I Bagchi et $\mathrm{al}^{14}$, Taiwo SS et $\mathrm{al}^{16}$, showing $75.2 \%, 66.7 \%$ sensitivity respectively.

Sensitivity pattern for Candida species was not tested because of non availability of test in our institute.

CAUTI cases were shown to have resistance to the penicillin group of antibiotics $(92.30 \%$ cases), Cephalosporin(90.38\%), Fluroquinolone(69.23\%) which is comparable to studies by Taiwo SS et $\mathrm{al}^{16}$, Dimri sanjeev et $\mathrm{al}^{13}$, Umesh kamat et $\mathrm{al}^{15}$, that showed $100 \%, 90.1 \%, 95.6 \%$ resistance respectively. 
Table 1: Incidence of CAUTI.

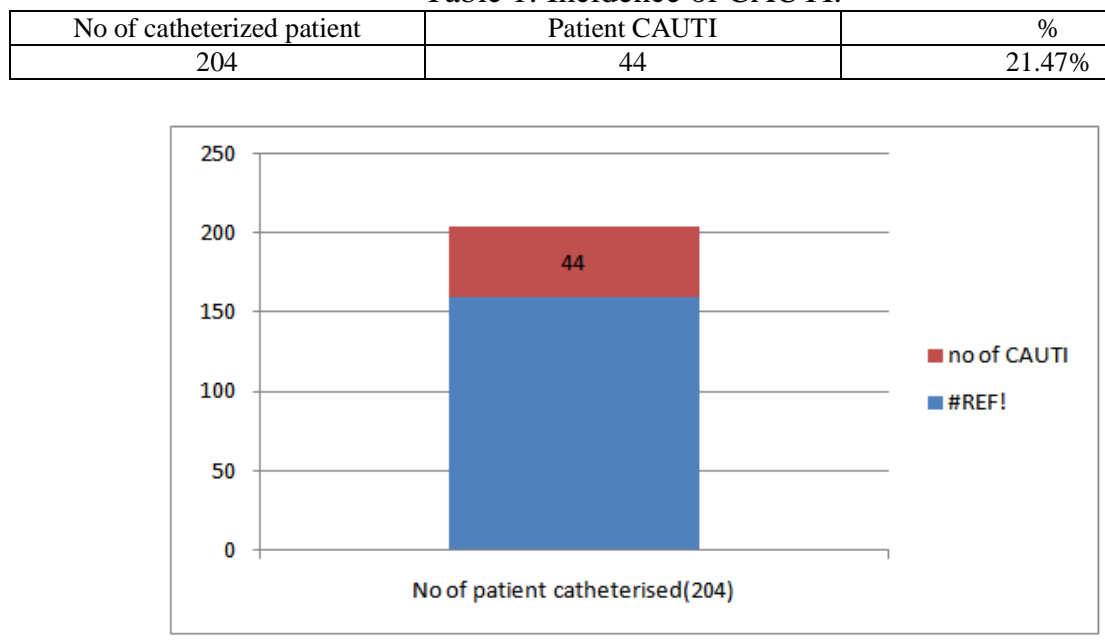

Table 2: Mean age and Gender wise distribution in total study population

\begin{tabular}{|l|c|c|c|c|}
\hline Gender & No. & Mean \pm SD age (yrs) & $\begin{array}{c}\text { Min. } \\
\text { Age }\end{array}$ & $\begin{array}{c}\text { Max. } \\
\text { Age }\end{array}$ \\
\hline Male & 133 & $49.02 \pm 17.96$ & 15 & 85 \\
\hline Female & 71 & $44.21 \pm 17.95$ & 15 & 80 \\
\hline Total & 204 & $46.48 \pm 17.82$ & 15 & 85 \\
\hline
\end{tabular}

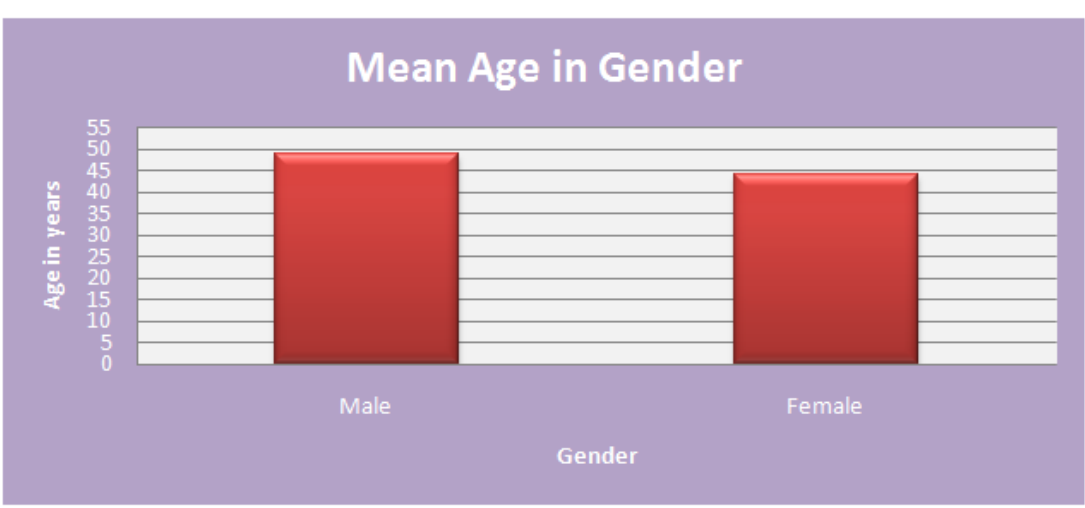

Table 3: Mean age and gender wise distribution of CAUTI patients:

\begin{tabular}{|l|c|c|}
\hline Gender & No. & Mean \pm SD age (yrs) \\
\hline Male & 30 & $48.17 \pm 14.34$ \\
\hline Female & 14 & $46.23 \pm 12.45$ \\
\hline Total & 44 & $48.98 \pm 16.34$ \\
\hline
\end{tabular}

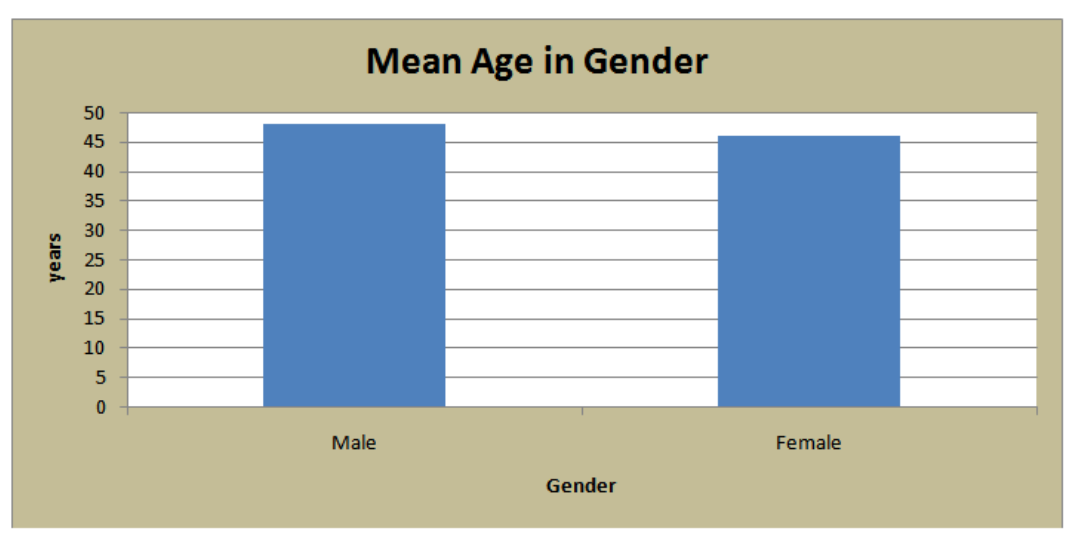


Table 4: Gender wise distribution of patients of CAUTI:

\begin{tabular}{|l|c|c|c|}
\hline \multirow{2}{*}{ CAUTI } & \multicolumn{2}{|c|}{ Sex } & \multirow{2}{*}{ Total } \\
\cline { 2 - 3 } & Male & Female & \\
\hline positive & $30(22.56 \%)$ & $14(19.72 \%)$ & $44(21.47 \%)$ \\
\hline Negative & $103(77.44 \%)$ & $57(80.28 \%)$ & $160(78.53 \%)$ \\
\hline Total & $133(100 \%)$ & $71(100 \%)$ & $204(100 \%)$ \\
\hline
\end{tabular}

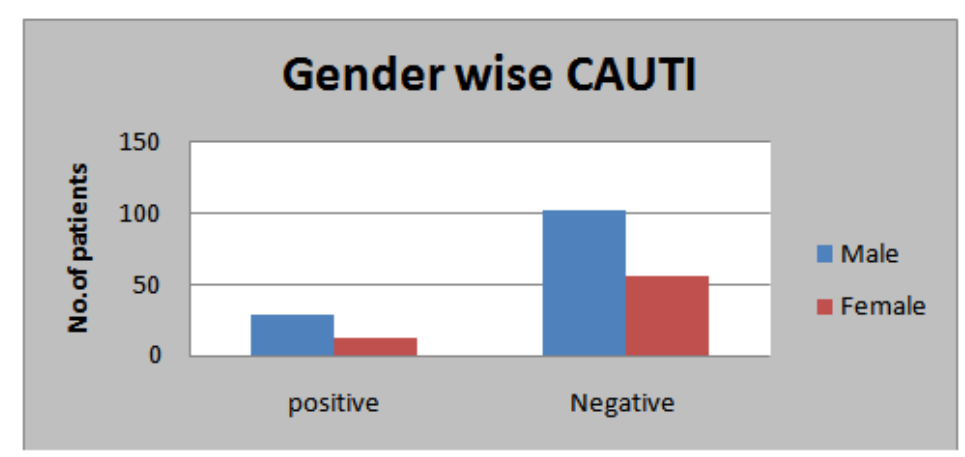

Table 5: Age wise distribution of patients of CAUTI:

\begin{tabular}{|l|c|c|c|}
\hline \multirow{2}{*}{ CAUTI } & \multicolumn{2}{|c|}{ Age-Group } & \multirow{2}{*}{ Total } \\
\cline { 2 - 4 } & $<40$ & $>40$ & $44(21.47 \%)$ \\
\hline Positive & $07(10.29 \%)$ & $37(27.20 \%)$ & $160(78.53 \%)$ \\
\hline Negative & $61(89.71 \%)$ & $99(72.80 \%)$ & $204(100 \%)$ \\
\hline Total & $68(100 \%)$ & $136(100 \%)$ & \\
\hline
\end{tabular}

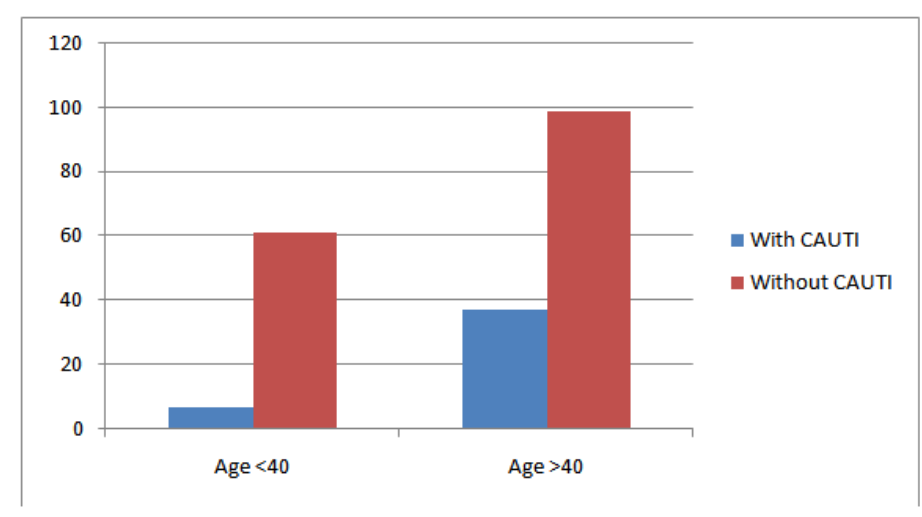

Table 6: Distribution of cases according to DIABETIC Mellitus:

\begin{tabular}{|l|l|l|l|}
\hline & CAUTI & Non CAUTI & Total \\
\hline Diabetic & $13(39.39 \%)$ & $20(60.60 \%)$ & $33(100 \%)$ \\
\hline Non Diabetic & $31(18.12 \%)$ & $140(81.87 \%)$ & $171(100 \%)$ \\
\hline Total & $44(21.47 \%)$ & $160(78.43 \%)$ & $204(100 \%)$ \\
\hline
\end{tabular}

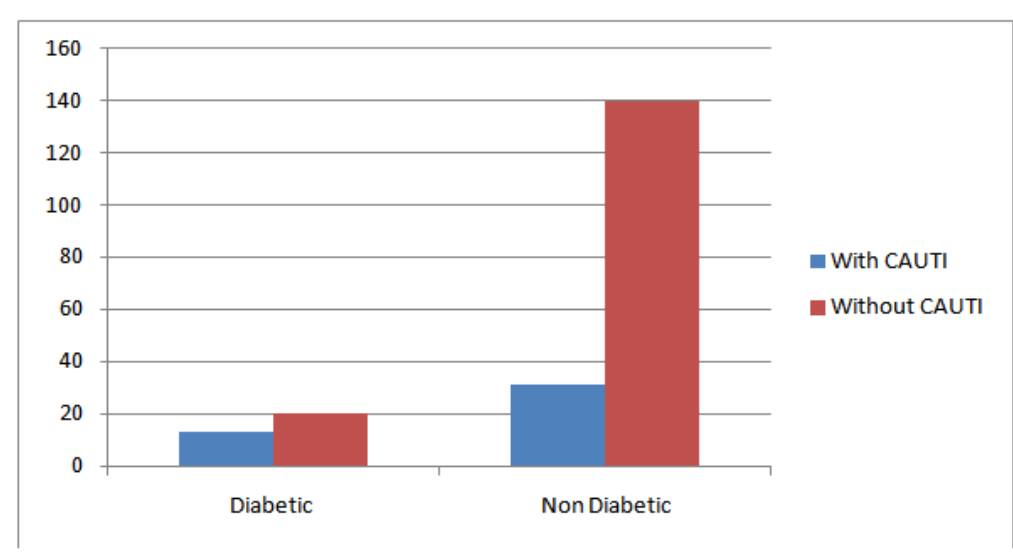


Table7: INCIDANCE OF CAUTI IN RENAL FAILURE

\begin{tabular}{|l|l|l|l|}
\hline & CAUTI & Non CAUTI & Total \\
\hline Renal failure & $22(50 \%)$ & $22(50 \%)$ & $44(100 \%)$ \\
\hline No Renal failure & $22(13.75 \%)$ & $138(86.25 \%)$ & $160(100 \%)$ \\
\hline Total & $44(21.55 \%)$ & $160(78.45 \%)$ & $204(100 \%)$ \\
\hline
\end{tabular}

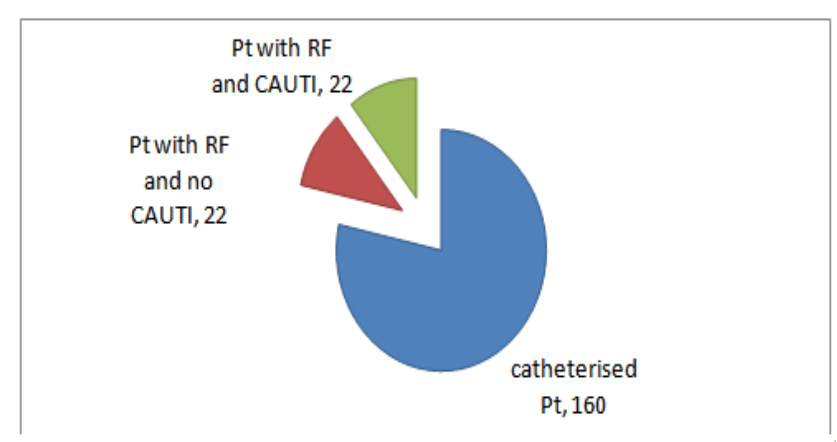

Table 8: INCIDANCE OF CAUTI AS PER DURATION OF CATHETERISATION

\begin{tabular}{|c|c|c|}
\hline Duration & Patient with CAUTI & $\%$ \\
\hline 3day & 15 & 34.90 \\
\hline 7day & 29 & 65.09 \\
\hline Total & 44 & 100 \\
\hline
\end{tabular}

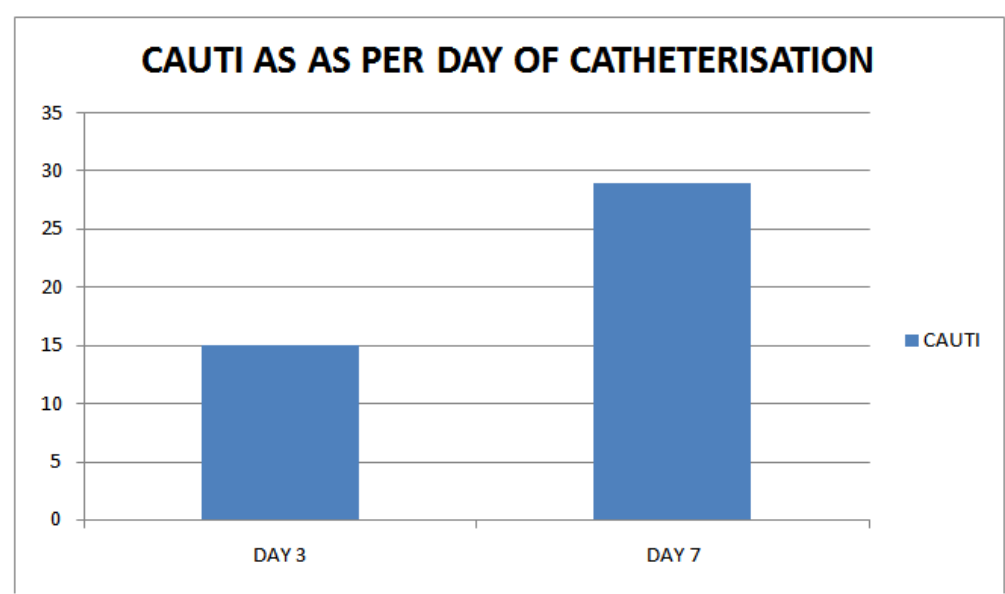

Table 9: Most common organisms.

\begin{tabular}{|l|c|c|}
\hline Commonest organism(s) (57) & No. & $\%$ \\
\hline E.coli & 27 & $47.36 \%$ \\
\hline Klebsiella & 11 & $19.20 \%$ \\
\hline Pseudomonas Aruginosa & 08 & $14.10 \%$ \\
\hline Candida & 05 & $8.70 \%$ \\
\hline Streptococci & 03 & $5.20 \%$ \\
\hline staphalococcus aureus & 02 & $3.50 \%$ \\
\hline Gm negative Bacilli & 01 & $1.75 \%$ \\
\hline
\end{tabular}




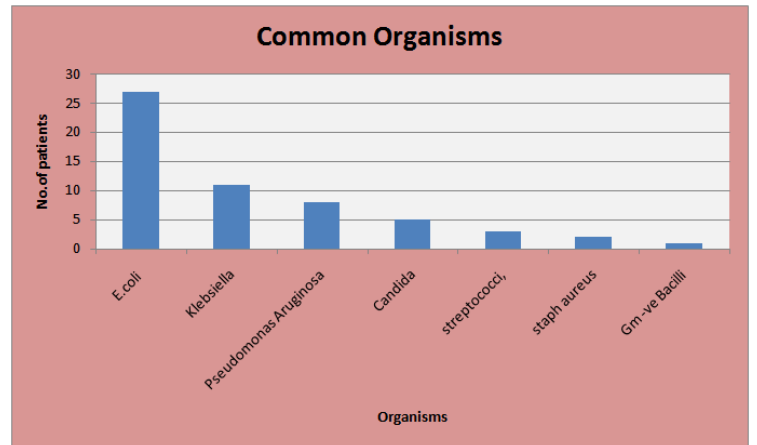

Table 10: Sensitivity of Drug for organisms.

\begin{tabular}{|c|c|c|c|c|c|c|c|c|c|}
\hline $\begin{array}{l}\text { organism } \\
\text { (s) }\end{array}$ & $\begin{array}{c}\text { AMOX+ } \\
\text { CLAV }\end{array}$ & $\begin{array}{c}\text { Cotrimo } \\
\text { xazole }\end{array}$ & $\begin{array}{c}\text { cephalos } \\
\text { porins }\end{array}$ & $\begin{array}{c}\text { Fluro } \\
\text { Quinolo } \\
\text { nes }\end{array}$ & $\begin{array}{c}\text { Linezoli } \\
\mathrm{d}\end{array}$ & $\begin{array}{c}\text { Nitrofur } \\
\text { ontoin }\end{array}$ & $\begin{array}{c}\text { Imepene } \\
\mathrm{m}\end{array}$ & $\begin{array}{l}\text { Piperacil } \\
\text { lin+ } \\
\text { tazobact } \\
\text { um }\end{array}$ & $\begin{array}{l}\text { Amikaci } \\
\mathrm{n}\end{array}$ \\
\hline $\begin{array}{c}\text { Ecoli } \\
(27)\end{array}$ & $\begin{array}{l}04 \\
(14.88 \%)\end{array}$ & $\begin{array}{c}05 \\
(18.51 \%)\end{array}$ & $\begin{array}{c}03 \\
(11.11 \%)\end{array}$ & $\begin{array}{c}01 \\
(3.70 \%)\end{array}$ & $\begin{array}{c}01 \\
(3.70 \%)\end{array}$ & $\begin{array}{c}16 \\
(59.25 \% \\
)\end{array}$ & $\begin{array}{c}15 \\
(55.55 \% \\
)\end{array}$ & $\begin{array}{c}16 \\
(59.25 \% \\
)\end{array}$ & $\begin{array}{c}18 \\
(66.66 \% \\
)\end{array}$ \\
\hline $\begin{array}{l}\text { Klebsiell } \\
\text { a } \\
(11)\end{array}$ & 00 & $\begin{array}{c}01 \\
(9.09 \%)\end{array}$ & 00 & $\begin{array}{c}02 \\
(18.18 \% \\
)\end{array}$ & 00 & $\begin{array}{c}05 \\
(45.45 \% \\
)\end{array}$ & $\begin{array}{c}09 \\
(81.81 \% \\
)\end{array}$ & $\begin{array}{c}06 \\
(54.54 \% \\
)\end{array}$ & $\begin{array}{c}05 \\
(45.45 \% \\
)\end{array}$ \\
\hline $\begin{array}{l}\text { Pseudom } \\
\text { onas } \\
\text { Aruginos } \\
\text { a(08) }\end{array}$ & 00 & $\begin{array}{c}01 \\
(12.5 \%)\end{array}$ & $\begin{array}{c}02 \\
(25 \%)\end{array}$ & $\begin{array}{c}02 \\
(25 \%)\end{array}$ & 00 & $\begin{array}{c}01 \\
(12.5 \%)\end{array}$ & $\begin{array}{c}04 \\
(50 \%)\end{array}$ & $\begin{array}{c}04 \\
(50 \%)\end{array}$ & $\begin{array}{c}05 \\
(62.5 \%)\end{array}$ \\
\hline $\begin{array}{l}\text { streptoco } \\
\text { cci,(03) }\end{array}$ & 00 & $\begin{array}{c}01 \\
(33.33 \%)\end{array}$ & 00 & 00 & 00 & $\begin{array}{l}01(33.33 \\
\%)\end{array}$ & $\begin{array}{l}01(33.33 \\
\%)\end{array}$ & $\begin{array}{l}01(33.33 \\
\%)\end{array}$ & $\begin{array}{c}03(100 \% \\
)\end{array}$ \\
\hline $\begin{array}{l}\text { staphaur } \\
\text { eus } \\
(02)\end{array}$ & 00 & $\begin{array}{c}01 \\
(50 \%)\end{array}$ & 00 & $\begin{array}{c}01 \\
(50 \%)\end{array}$ & $\begin{array}{c}01 \\
(50 \%)\end{array}$ & 00 & 00 & 00 & $\begin{array}{c}02 \\
(100 \%)\end{array}$ \\
\hline $\begin{array}{l}\text { Gm-ve } \\
\text { Bacilli, } \\
(01)\end{array}$ & 00 & 00 & 00 & 00 & 00 & $\begin{array}{c}01(100 \% \\
)\end{array}$ & $\begin{array}{c}01(100 \% \\
)\end{array}$ & $\begin{array}{c}01(100 \% \\
)\end{array}$ & $\begin{array}{c}01(100 \% \\
)\end{array}$ \\
\hline
\end{tabular}

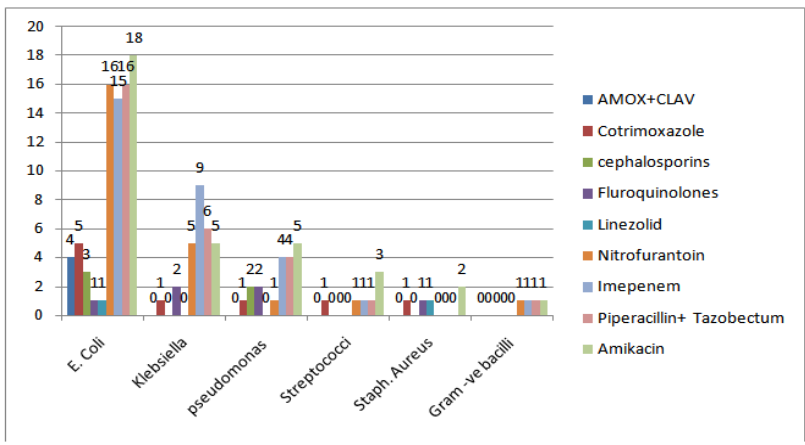

Table 11: Resistance of Drug for organisms.

\begin{tabular}{|c|c|c|c|c|c|c|c|c|c|}
\hline organism(s) & $\begin{array}{l}\text { AMOX+ } \\
\text { CLAV }\end{array}$ & $\begin{array}{c}\text { Cotrimoxazol } \\
\mathrm{e}\end{array}$ & $\begin{array}{c}\text { cephalosporin } \\
\text { s }\end{array}$ & Fluro Quinolone & Linezolid & $\begin{array}{l}\text { Nitrofuront } \\
\text { oin }\end{array}$ & Imepenem & $\begin{array}{l}\text { Piperacillin } \\
+ \\
\text { tazobactum }\end{array}$ & Amikacin \\
\hline $\begin{array}{c}\text { Ecoli } \\
(27)\end{array}$ & $23(85.18 \%)$ & $22(81.48 \%)$ & $24(88.88 \%)$ & $16(59.25 \%)$ & $26(96.29 \%)$ & $11(40.74 \%)$ & $\begin{array}{c}12(44.44 \\
\%) \\
\end{array}$ & $\begin{array}{c}11(40.74 \% \\
)\end{array}$ & $09(33.33 \%)$ \\
\hline $\begin{array}{l}\text { Klebsiella } \\
(11)\end{array}$ & 11(100\%) & $10(99.9 \%)$ & $11(100 \%)$ & $09(81.8 \%)$ & $11(100 \%)$ & $06(54.5 \%)$ & $02(18.2 \%)$ & $05(45.4 \%)$ & $06(54.5 \%)$ \\
\hline $\begin{array}{l}\text { PseudmonArug } \\
\text { inosa(8) }\end{array}$ & $08(100 \%)$ & $07(87.5 \%)$ & $06(75 \%)$ & $06(75 \%)$ & $08(100 \%)$ & $07(87.5 \%)$ & $04(50 \%)$ & $04(50 \%)$ & $03(37.5 \%)$ \\
\hline $\begin{array}{l}\text { streptococci, }(0 \\
3)\end{array}$ & $03(100 \%)$ & $02(66.66 \%)$ & $03(100 \%)$ & $03(100 \%)$ & $03(100 \%)$ & $02(66.66 \%)$ & $\begin{array}{l}02(66.66 \\
\%)\end{array}$ & $02(66.66 \%$ & 00 \\
\hline $\begin{array}{l}\text { staphaureus(02 } \\
\text { ) }\end{array}$ & $02(100 \%)$ & 01(50\%) & $02(100 \%)$ & $01(50 \%)$ & $01(50 \%)$ & $02(100 \%)$ & $02(100 \%)$ & $02(100 \%)$ & 00 \\
\hline $\begin{array}{l}\text { Gm-ve } \\
\text { bacilli,(1 }\end{array}$ & $01(100 \%)$ & $01(100 \%)$ & $01(100 \%)$ & $01(100 \%)$ & $01(100 \%)$ & 00 & 00 & 00 & 00 \\
\hline
\end{tabular}




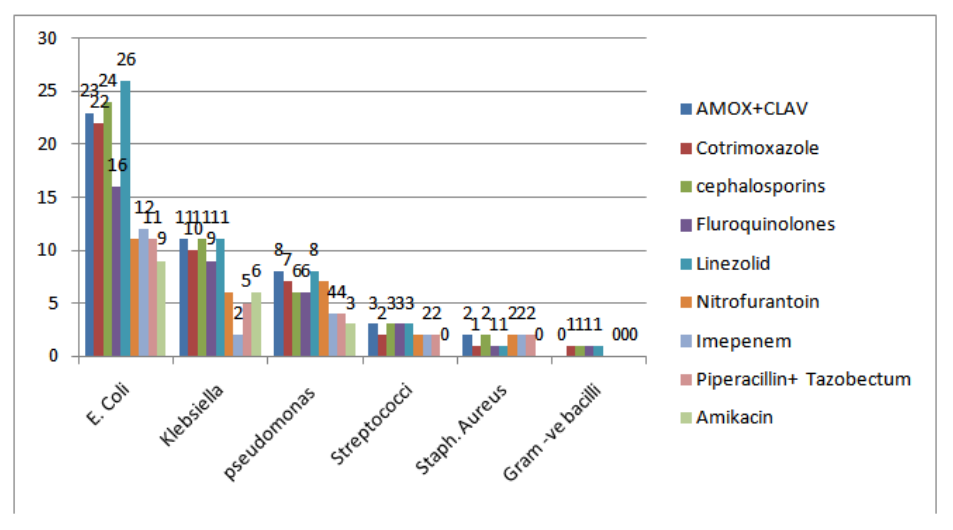

\section{Conclusion}

The incidence of CAUTI in MGM MCH, Aurangabad was 21.47\%.Male patients were found to be more susceptible to CAUTIs.Age above 40 years was found as important risk factor for CAUTI.Patient with comorbidities like Diabetes Mellitus and Renal failure found at increased risk of CAUTI.As Duration catheterization increases incidence of CAUTI increases.E coli were the most common organism isolated from CAUTI cases in our ICU setting.CAUTI patients were most susceptible to Amikacin, Imepenem and piperacillin+Tazobactum in our study.High resistance was found with Penicillin, cephalosporin, fluroquinolones group of antibiotic in CAUTI patients

[1] Richards mj, edwards jr , culver dh, gaynes rp: nosocomial infections in combined medical-surgical intensive care units in the united states, infect control hosp epidemiol 2000, 21: 510-515.

[2] Laupland $\mathrm{kb}$, zygun da, davies hd, church $\mathrm{dl}$, louie tj, doig cj: incidence and risk factors for acquiring nosocomial urinary tract infection in the critically ill, j crit care 2002; 17: 50-57.

[3] Laupland $\mathrm{kb}$, bagshaw sm, gregson $\mathrm{db}$, kirkpatrick aw, rosset, church $\mathrm{dl}$ : intensive care unit -acquired urinary tract infections in a regional critical care system,critical care ,2005,9:60-65.

[4] Schaeffer aj: catheter-associated bacteriuria, urol clin north am, 1986 nov; 13(4):735-747

[5] Leone M, Albanese J, Garnier F, et al: Risk factors of nosocomial catheter-associated urinary tract infection in a polyvalent intensive care unit. Intensive Care Med. 2003; 29(7):929-932.

[6] Stamm we, catheter-associated urinary tract infections: epidemiology, pathogenesis, and prevention. American j of med, 1991; 91(3b) 65s-71s.

[7] Trautner BW, Darouche RO: Catheter-associated infections. Arch Intern Med, April 2004; 164: 842-850

[8] Stark RP, Maki DG: Bacteriuria in a catheterized patient. N Eng J Med 1984; 311:560-4.

[9] Yonit Wiener-Well,Ina Gofman , Marc V. Assous, Yossi Freier-Dror,Amos M. Yinnon, Tamar Lachish. The clinical significance of isolation of two different organisms from the urine of patients with an indwelling catheter. Diagnostic Microbiology and Infectious Disease (2013)

[10] Tissot E, Limat S, Cornette C, Capellier G: Risk factors for catheterassociated bacteriuria in a medical intensive care unit.Eur J Clin Microbiol Infect Dis 2001; 20:260-262.

[11] Bhatia N, Daga MK, Garg S, Prakash SK: Urinary catheterization in medical wards J Glob Infect Dis. 2010 May; 2(2):83-90.

[12] Joon Ho Lee, Sun Wook Kim, Byung Il Yoon, U-Syn Ha, Dong Wan Sohn, Yong-Hyun : Factors That Affect Nosocomial CatheterAssociated Urinary Tract Infection in Intensive Care Units: 2-Year Experience at a Single Center. ChoKorean J Urol 2013; 54:5965.

[13] Dimri Sanjeev, Sharma Hemant, Datta S K, Gupta Deepak :A Study of Urinary Tract Infections in Patients with Catheter in Tertiary Care Hospital in Western Uttar Pradesh International Journal of Contemporary Surgery Year : 2013, Volume : 1, Issue : 2,83-89.

[14] Dr. Indranil Bagchi, Dr. Neelam K Jaitly,Dr. V.R.Thombare :MICROBIOLOGICAL EVALUATION OF CATHETER ASSOCIATED URINARY TRACT INFECTION IN A TERTIARY CARE HOSPITAL.International Journal of Biological and Health Science, Vol.1 Issue II Jan. 2013(01-10).

[15] Umesh S. Kamat, Agnelo Fereirra,Dilip Amonkar,Dilip D. Motghare and Manoj S. Kulkarni:Epidemiology of hospital acquired urinary tract infections in a medical college hospital in Goa Indian J Urol. 2009 Jan-Mar; 25(1): 76-80.

[16] Taiwo SS and 2Aderounmu AOA:Catheter Associated Urinary Tract Infection: Aetiologic Agents and Antimicrobial Susceptibility Pattern in Ladoke Akintola University Teaching Hospital, Osogbo, Nigeria. African Journal of Biomedical Research, Vol. 9 (2006); $141-148$.

[17] Namita Jaggi and Pushpa Sissodia: MULTI DIMENTIONAL SUPERVISION PROGRAME TO REDUCE CAUTI AND ITS ANALYSIS TO ANABLE FOCUS ON LABOUR AND COST EFFECTIVE INFECTION CONTROL IN A TERTIARY CARE HOSPITAL. J Clin Diagn Res. 2012 October; 6(8): 1372-1376.

[18] Dogru A et al: The Rate of Device-Associated Nosocomial Infections in a Medical Surgical Intensive Care Unit of a Training and Research Hospital in Turkey: One-Year Outcomes. Jpn. J.Infect. Dis., 2010; 63: 95-98.

[19] Sallam SA, Arafa MA, Razek AA, Naga M, Hamid MA:Nosocomial infections. Eastern Mediterranean Journal, 2005; 11(1/2): 5361. 